\title{
HOW TO EXECUTE ROBUST DESIGN OPTIMIZATION
}

\author{
Igor N. Egorov, Gennadiy V. Kretinin and Igor A. Leshchenko \\ IOSO Technology Center, Moscow, Russia
}

\begin{abstract}
The problem of robust design optimization consists in the search for technical solutions that can be implemented in practice with high probability. From mathematical point of view the problem comes to optimization of design parameters of the system under investigation according to probabilistic criteria. This paper presents essential stages of robust design optimization solution: identifying uncertainties and choosing mathematical model; forming of probabilistic criteria; optimization problem statement; choosing the procedure for calculation of probabilistic criteria and choosing the optimization technique. Options for robust design optimization solution are considered with the use of multicriteria optimization techniques as well as with procedures of multilevel optimizations. Examples of real-life problems solution are also given.
\end{abstract}

\section{Introduction}

Designing a complex technical system in present-day conditions is impossible without the use of optimization techniques. In fact, design and optimization processes do represent a single whole. As soon as humans started to create technical devices (stone axes, for example), they thought about making their tools as efficient as possible. At first, people used to design and optimize technical systems simultaneously, as they thought about the devices they make. While designing a technical system and picking up its parameters the designer had always been implicitly assessing possibilities of practical implementation of the system.

The rise of the complexity of systems as well as the number of parameters needed to be coordinated with each other in an optimal way have led to the necessity of using mathematical modeling of systems and application of optimization techniques. In this situation the designer focuses on working out of an adequate mathematical model and the analysis of the results obtained. Choosing optimal parameters for the system being designed is done through the use of formal mathematical optimization procedures. The use of such an approach exempts the designer of routine work aimed to select optimal combinations of variable parameters, allowing him to set and solve extremely complex problems of optimal designing. However, solutions obtained by means of mathematical modeling and optimization techniques in most cases are hard to implement in real life. This is largely due to the fact that while stating and solving optimization tasks by traditional (deterministic) approach, as a rule, various uncertainties influencing the efficiency of the designed system in real life conditions are not taken into consideration.

An efficiency extremum value, obtained during the optimization problem solving in a deterministic setting, is a maximum attainable value and can be considered as just conventional optimum from the point of view of its practical implementation. Based on this fact, Jacek Marczyk states ${ }^{1}$ : "Optimization is actually just the opposite of robustness". He considers the stochastic simulation using the Monte-Carlo technique as an alternative to traditional optimization. Our experience in the field of real-life optimization allows us to reformulate Marczyk's thesis in the following form: "Deterministic optimization is actually just the opposite of robustness". Since the $1992^{2}$ we are speaking about the necessity to use the "stochastic optimization" vs. "deterministic optimization" for real-life problems.

In recent years, probabilistic design analysis and optimization methods have been developed ${ }^{3,5,6,8-10}$ to account for uncertainty and randomness through stochastic simulation and probabilistic analysis. Totality of such methods can be treated as the new scientific direction, named "Robust Design Optimization" (RDO). The distinct feature of this direction is the use of probability criteria to evaluate the technical system quality. The current paper does not provide a full review of literature in this growing field, but does include selected papers, characterizing the most interesting features of this approach.

When solving problems of this class authors concentrate on the following main goals:

1. Identify designs, which minimize the mean value of the performance under uncertain manufacturing conditions;

2. Identify designs, which minimize the variability of the performance under uncertain (manufacturing or operation) conditions;

3. Provide the best overall performance over the long time exploitation of the system;

4. Provide the best probability to ensure the preset constraints;

\section{Various combinations of the goals $1-4$.}

Despite great variety of problem statements and the methods to solve optimization problems in conditions of uncertainty, there are a number of common problems that should be addressed by the investigators. In this paper we will try to indicate these problems and consider some ways for their solution. 


\section{Identifying uncertainties, choosing mathematical model}

A complex technical system cannot be created efficiently without a correct mathematical formalization of the design problem. While creating a system the designers are to form the vector of values of system efficiency $y=\left(y_{1}, y_{2}, \ldots, y_{m}\right)$, which are to be maximized, minimized and constrained, to form the vector of variable parameters $x=\left(x_{1}, x_{2}, \ldots, x_{n}\right)$, varying of which leads to the variation of the efficiency, and to form the vector of external conditions $e=\left(e_{1}, e_{2}, \ldots, e_{k}\right)$. Correlation between these vectors as $y=f(x, e)$ forms the mathematical model of the system under investigation. Existence of mathematical model makes it possible to formulate a design problem as an optimization task, which lies in the search of one or several vectors $x^{*} \in D$ that ensure the best (in some way) efficiency. Here

$$
\begin{aligned}
& D=\left\{x \in R^{n} \mid x_{j-} \leq x_{j} \leq x_{j+}, j=\overline{1, n} ;\right. \\
& \left.g_{i}(x, e) \leq 0, i=\overline{1, w}\right\}-
\end{aligned}
$$

is the search region, $g_{i}(x, e)$ is the constrained efficiency values. Such an "ideal" design problem statement was regarded, until recently, as a necessary and sufficient condition for obtaining an optimal design. In practice, however, such an approach to solving real-life tasks deals with serious problems connected with impossibility to implement optimal project solutions. The main reason for this lies in existence of a large number of uncertainties, which are not taken into account while modeling of the system, optimization problem statement and solving.

Generally, system operation is affected by uncertainties in geometrical parameters due to production technology spread; in materials properties; in mathematical model accuracy; in environment conditions, etc. The recent work by Batill, et.al. ${ }^{1}$ presents the number of issues related to the various types of uncertainty in multidisciplinary design optimization. Identification of uncertainties to be considered in every specific case is defined by physical problem statement as well as the possibilities of the mathematical model utilized.

The attempt to include uncertainties while design problem formalization results in the necessity to consider relations: $x=x\left(\bar{x}, \xi_{x}\right) ; e=e\left(\bar{e}, \xi_{e}\right)$; $f(x, e)=\Psi\left(\bar{f}(x, e), \xi_{f}(x, e)\right)$, where $\bar{x}, \bar{e}, \bar{f}$ are ideal vectors of variable parameters, environmental conditions and the ideal mathematical model; $\xi=\left(\xi_{x}, \xi_{e}, \xi_{f}\right)$ is the vector of random values including uncertainties in implementation of variable parameters, environment conditions and the mathematical model accuracy. Generally, to solve a RDO problem one must be able to determine the system efficiency values $y=f(x, e)$ for given values of $\bar{x}, \bar{e}$, and hence to know the laws of distribution of $\xi$ vector components and functional dependence of $\Psi\left(\bar{f}, \xi_{f}\right)$. To illustrate this task let us consider some of typical cases.

\section{Including uncertainties in variable parameters values}

This task generally occurs while optimization of design parameters of the system for given level of production technology, when it is necessary to ensure either maximum of mean value of efficiency or minimum of deviation of efficiency from the average value in mass production. In this case, to determine the laws of distribution of components of $\xi_{x}$ vector either a detailed analysis of technological processes can be used, or statistical data accumulated during production of similar systems. However, in most cases, components of the vector $\xi_{x}$ submit to a normal or a close to normal distribution.

\section{Including uncertainties of environment conditions}

The problem of including uncertainties of environment conditions is usually formulated as an adaptation task, where functioning of a technical system is being adjusted to changing environment by moving adjustable controls. For non-regulated conditions, however, or for systems without possibilities of measuring environment parameters, the Robust Design Optimization problem should be solved to minimize the influence of uncertainty of environment conditions on the efficiency values. In this case, the laws of distribution of components of $\xi_{x}$ vector are necessary. These laws, as a rule, represent results of integrating of experimental data.

It is necessary to note that to fulfill optimization that incorporates uncertainties of variable parameters and the environment conditions a conventional deterministic mathematical model can be used as $y=\bar{f}\left(\bar{x}, \bar{e}, \xi_{x}, \xi_{e}\right)$.

\section{Including accuracy of mathematical model}

The necessity to include accuracy of mathematic modeling most often comes during solving of design problem of perspective system when modeling is based on some assumptions about attainability of some of the properties of materials, efficiency of different subsystems, forecasts of the market situation. In this case the problem is formulated as the problem of maximization of probability of attainment of some of the values of efficiency. Determining of distribution laws for components of vector $\xi_{f}$ and 
the functional dependence of $\Psi\left(\bar{f}, \xi_{f}\right)$ is done in this case based on existing statistical dependencies and expert assessments.

\section{Probabilistic criteria}

When solving robust design optimization the efficiency values $y=f(x, e)$ are random ones. In this case it is necessary to use probabilistic optimization criteria $\tilde{y}(\bar{x})$. Let us consider some of the probabilistic criteria used when the efficiency index $y(x, e)$ is to be minimized.

1. $\tilde{y}(\bar{x})=M\{f(x, e)\}$ - mean value of efficiency;

2. $\tilde{y}(\bar{x})=\sigma\{f(x, e)\}$ - magnitude of efficiency value deviation;

3. $\tilde{y}(\bar{x})=P_{c}\left\{f(x, e) \leq y_{p}\right\}$ - probability that efficiency value is no worse than the one given;

4. $P_{c}\{f(x, e) \leq \tilde{y}(\bar{x})\} \geq P_{p}$ - efficiency value ensured with probability no less than the one given.

Each of these criteria reflects different robust properties of the project. Pic. 1 shows a sample graph of function $y=(x-1)^{2} \cdot\left((x-5)^{4}+1\right)$ and probabilistic criteria No.1,2 for the case of $x=\bar{x}(1+\xi)$, where $\xi$ is a normally distributed random value with average of distribution equaling 0 , and dispersion equaling 0.3 .

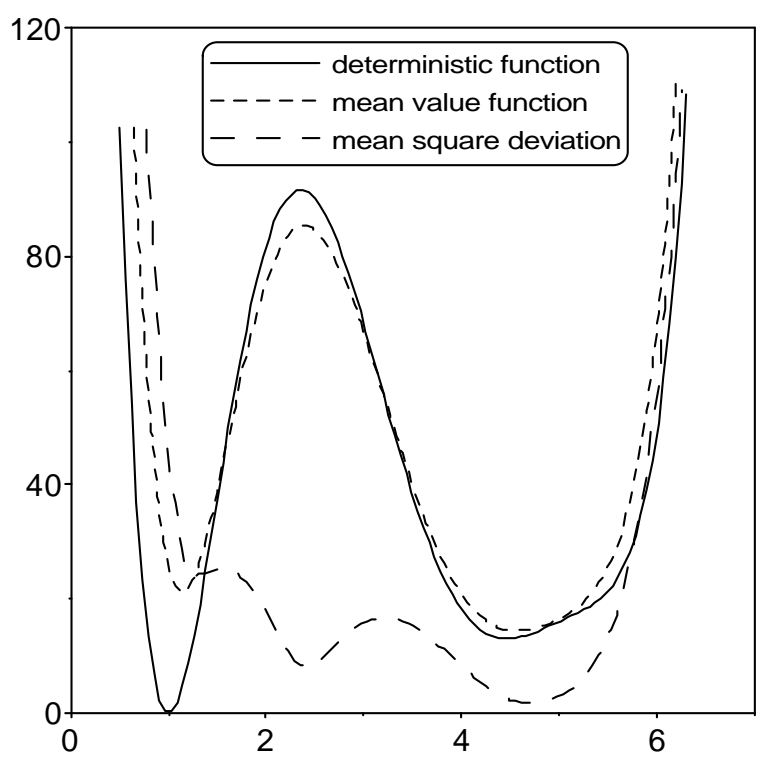

Fig. 1 Examples of probability criteria.

Suppose this equation represents some mathematical model of a system where efficiency index $y$ is to be minimized. It is obvious that the minimum of given function (deterministic solution) is reached at $x=1$.
If supposed that the design parameter $x$ is a random value depending on, say, technological spread, than minimum of the average value of efficiency (probabilistic criterion No.1) is reached as early as $\bar{x} \approx 4.6$. Location of minimum by probabilistic criterion No.2 also differs from the deterministic solution. The considered example leads to an important conclusion that an RDO problem should not be reduced to the problem of "correction" of solution obtained through deterministic approach since extremums of probabilistic criteria may substantially differ, by design parameters, from extremum of deterministic criterion. This in fact means that in vicinity of deterministic solution there might be a solution improving robust properties of the project, however, to achieve maximal robust properties the problem must be solved by probabilistic criteria.

It should be noticed that in the case of robust design optimization all of the constrained parameters of the system $g_{j}(x, e) \leq 0, j=\overline{1, w}$ are also random values, and it is necessary to evaluate their probabilistic criteria. To simplify procedures of optimization problem solution and analysis of the obtained results some integral value can be introduced. This value is the probability of observing of all given constraints $-P_{o}(\bar{x})=P(x \in D)$. Thus, from mathematical point of view the problem of robust design optimization can be formulated as follows: for given laws of distribution of components of vector $\xi$ a vector $\bar{x}^{*}$ is to be found that will ensure $\tilde{y}\left(\bar{x}^{*}\right)=\operatorname{extr}\{\tilde{y}(\bar{x})\}$, where $P_{0 p}$ is the given probability of constraints observation.

Besides, value $P_{0}(\bar{x})$ may be regarded as both additional criterion while stochastic optimization and can be used to calculate other criteria, for example:

5. $\tilde{y}(\bar{x})=P_{\Sigma}\left\{f(x, e) \leq y_{p}\right\}$ - integrated probability that the value of efficiency is not worse than a given one $\left(P_{\Sigma}=P_{c} \cdot P_{0}\right)$.

6. $P_{\Sigma}\{f(x, e) \leq \tilde{y}(\bar{x})\} \geq P_{p}$ - value of efficiency ensured with integrated probability no less than a given one $\left(P_{\Sigma}=P_{c} \cdot P_{0}\right)$.

Selecting this or that probabilistic criterion is determined by specific features of the optimization task. At the same time it is necessary to take into account that when applying criteria No.3...No.6, extra research is to be done to set correctly values of $P_{p}$ and $y_{p}$, since in this case a situation with no solution to the set problem may occur.

It is quite obvious that the formulated probabilistic criteria can contradict one another. Thus, for instance, 
it will be sufficient to simply ensure high robust properties for a project (high values for $P_{c}, P_{0}, P_{\Sigma}$ ) by artificially decreasing requirements for efficiency value $y_{p}$. Hence, robust design optimization problems (even for a single chosen efficiency) are in essence multicriteria ones and appropriate techniques to solve them should be used.

\section{Caclulating probabilistic criteria, choosing optimization technique}

The main problem occurring while solving robust design optimization problem is determining of probabilistic criteria values. There are various approaches to solve this problem.

The most reliable information about values of probabilistic criteria can be obtained by means of analytical approach. In this case for every fixed $\bar{x} \in D$ while determining, for instance, probability $P_{c}\left\{f(x, e) \leq y_{p}\right\}$, calculation of multidimensional integral $\int_{\xi \in \Omega} P_{c}(\bar{x}, \bar{e}, \xi) d \xi$ is required. This problem is a quite complicated one if dimensionality $n>2$. Bearing in mind that for complex technical systems it is practically impossible to analytically estimate the laws of distribution of efficiency and constraints (which is repeatedly required during iterative search for extremum), difficulties of solving real world tasks with this approach become clear.

The simplest and the most universal method of evaluation of probabilistic criteria is the Monte-Carlo method. The main advantage of this method, as applied to RDO problems, is no necessity of setting of any a priori assumptions about the goal function peculiarities (smoothness, monotony, continuity, differentiability, and so forth). However, efficiency of Monte-Carlo method when solving real life problems to a great extent depends on the required accuracy of definition of probabilistic criteria. Particularly, applying gradient methods of optimization, requiring high accuracy of definition of probabilistic criteria, results in high computational expense (required number of tests at each iteration of extremum search makes up $\approx 10^{6} \ldots 10^{9}$ ).

Second approach includes a number of methods which are based on different approximation techniques (Taylor's series, response surfaces and so on). An examples of these methods one can see in the work $^{10}$. The approximate methods of probability indexes evaluation usually require an additional information from mathematical model such as: responses sensitivities; variable to variable dependencies etc. Moreover, these methods are very sensitive with respect to topological peculiarities of objective functions and constraints.

Note, that any optimization algorithm realizes the iterative procedure with the large number of objective functions and constraints evaluations. Total time of solution of any optimization problem, an RDO problem in particular, can be defined as time of calculation of criteria for one value of variable parameters multiplied by the necessary number of such calculations $T_{\Sigma}=t_{c r} \cdot N_{\text {calc }}$. This simple formula indicates a great importance of choosing an appropriate optimization technique. Attempts to decrease the number of calculations of values of probabilistic criteria $N_{\text {calc }}$ leads to the necessity to use "fast" gradient methods of optimizations. However, efficiency of gradient methods substantially decreases when there is a "noisy" object function under investigation. Hence their usage requires high accuracy of assessment of probabilistic criteria, which, in its turn, leads to substantial increase of $t_{c r}$. Besides, the use of gradient techniques places substantial restrictions on topology of object functions, hence limiting their applicability when solving practical problems. A much more advantageous in this case can be the use of direct optimization techniques, having higher noise immunity and allowing for substantial decrease of the time $t_{c r}$ under minor increase of $N_{\text {calc }}$.

When solving real-life RDO problems we use algorithms of IOSO technology (Indirect Optimization on the basis of Self-Organization). High noise-proof features of these algorithms enables us to solve RDO problems by means of Monte-Carlo technique with extremely small amount of statistical tests at each of the search iteration. Figure 2 shows example of efficiency of IOSO algorithms when solving problems optimization for "noisy" object functions. It is evident that even under intensive noise IOSO algorithms reliably provide extremum region. With the use of such an approach a more accurate assessment of probabilistic criteria is done after the solution of optimization problem at the stage of analysis of the results obtained.

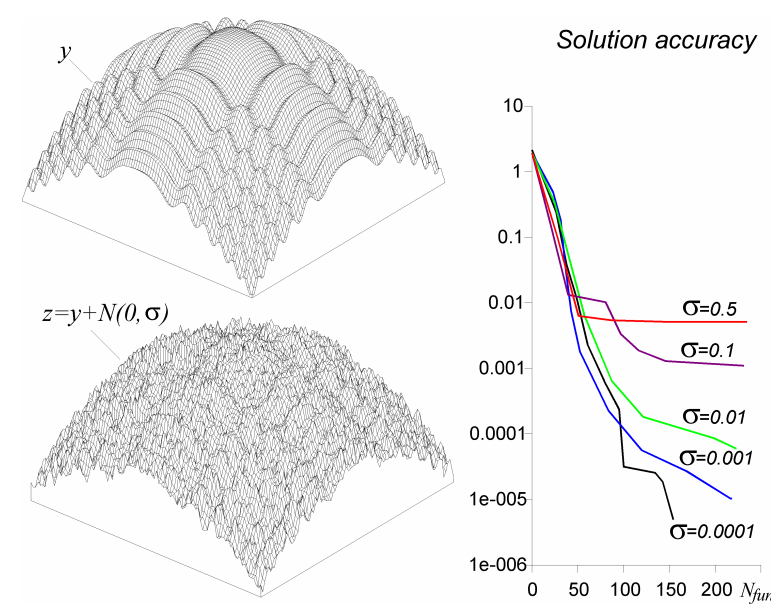

Fig. 2 Example of the IOSO algorithms noiseimmunity. 


\section{Multiple criteria in RDO problems}

The problem of reasonable choice of the procedure for calculation of probabilistic criteria as well as the optimization technique becomes more complicated if RDO problem assumes multiple criteria. As mentioned above, RDO problems are multicriteria ones by nature since they, in fact, assume compromises between what can be implemented in real life and the probability of achieving of obtained results. In these conditions the ultimate choice of the project for the investigated system is to be made based on the analysis of totality of Pareto-optimal projects, obtained with probabilistic and deterministic criteria.

Within the framework of IOSO technology we have elaborated a number of multicreteria algorithms to solve such complex problems. The main advantages of these algorithms over traditional mathematical programming approaches are the following ${ }^{3}$ :

- convolution approaches are not used in solving multiobjective problems;

- the algorithms determine the desired number of Pareto-optimal solutions, so that these solutions are uniformly distributed in the space of objective functions;

- it is possible to solve the optimization problems for the objective functions of complex topology: nonconvex, non-differentiable, with many local optima;

- relatively small number of probability indexes evaluations;

- it is possible to naturally employ the parallelization of the computational process.

These advantages are the basis for the wide use of the proposed method in the real-life problems.

Let us consider the example of the multiobjective robust design optimization of the multistage axial flow compressor. The brief description of this optimization problem:

- Purpose: To insure the maximum efficiency and maximum implementation probability under preset level of production technology;

- Setting features: 140 independent variables (flowpath geometry); two objectives; three nonlinear constraints (mass flow, pressure ratio, surge margin);

- Optimization process features: Object under study - quasi-3D mathematical model. Implementation probability was calculated as the probability of assuring the preset constraints.

Fig. 3 shows the main results of this problem. One can see that there is a compromise area between the ideal (deterministic) compressor efficiency and the implementation probability. In general, designer can select any solution from the obtained set. In this case the design No 4 was selected as the final design.
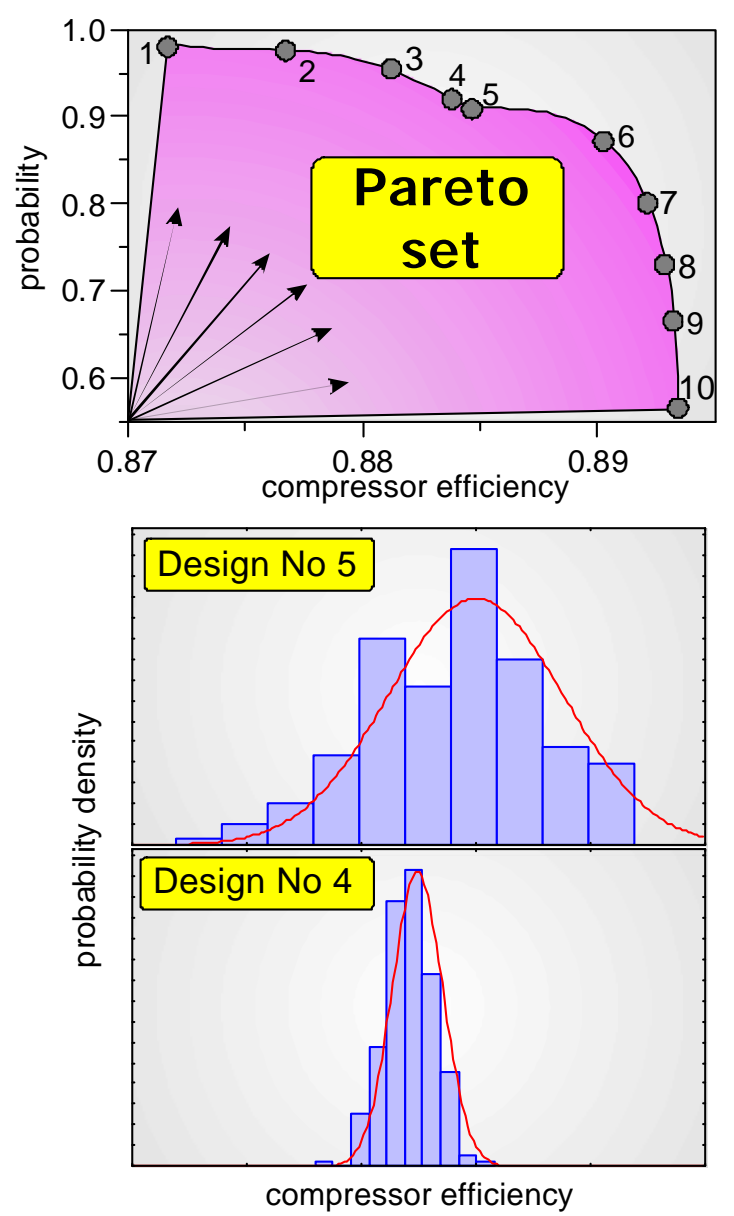

Fig. 3 Results of compressor multicriteria robust design optimization.

While solving this problem we used only 50 calls of mathematical model to approximately evaluate the probability criterion at each iteration. After 400 iterations optimization process was halted. Then we used 5000 additional calls of mathematical model to refine probability criteria for Pareto set found. Thus, total number of mathematical model calls was 25000 . This is a not enormous value for the optimization problem having 140 independent variables, but solution of the same problem using more accurate mathematical model is problematic one. In this case reasonable choice is the usage of so-called multilevel RDO procedure.

\section{Multilevel RDO}

The feature of the Multilevel Robust Design Optimization procedure is the use of mathematical models of various fidelity (from the lowest to the highest) during the solution process and adaptive switching between them ${ }^{4}$. This procedure provides minimization of the number of times the high fidelity (true) models are used without reducing the accuracy of the resulting solution (fig. 4). 


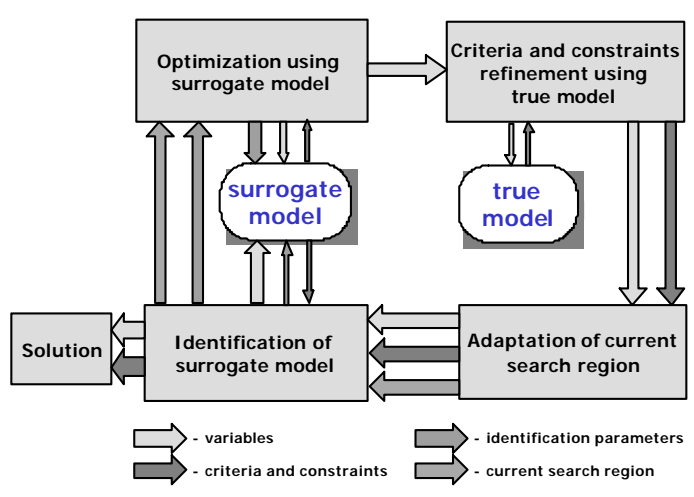

Fig. 4 Multilevel optimization scheme.

The efficiency of this procedure may be demonstrated using the similar compressor optimization problem with 63 independent variables (fig. 5). As the surrogate model we used the simplified axissymmetrical model which is possible to be identified. The main idea of multilevel optimization is that information stored during the search is used to improve the surrogate model. However, this identification is correct not for the entire initial search area but only for certain neighborhood of the obtained Pareto set. This ensures purposeful improvement of probability criteria evaluation accuracy only in the area of optimal solutions.

For this problem we obtained 10 Pareto-optimal solutions using only 60 direct calls to high-fidelity model. This example shows that it is possible to solve the optimization problem when the number of times the highest fidelity model is involved is less than the number of design variables. This provides considerable (several orders of magnitude) reduction in CPU time required for solution of complex optimization problems.

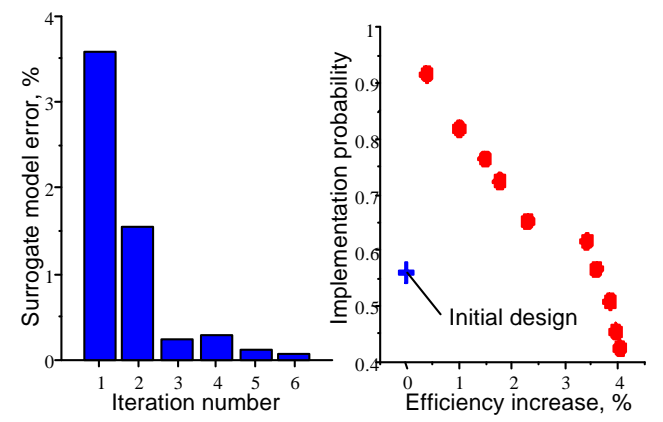

Fig. 5 Results of compressor multilevel robust design optimization.

\section{Conclusion}

The RDO problem is reduced to the problem of solution of optimization of the system's design parameters according to probabilistic criteria. The efficiency of solution of such problems depends absolutely on the chosen technique for calculation of deterministic criteria as well as the optimization technique. One of the most promising techniques to solve of RDO problems is usage of approximate assessments of probabilistic criteria under MonteCarlo combined with direct optimization techniques, IOSO technology algorithms, for example. When solving complex real life RDO problems, application of multilevel optimization procedures, worked our under IOSO technology, may turn out an extremely effective way.

\section{References}

[1] S. M. Batill, J.E. Renaud and Xiaoyu Gu, Modeling and Simulation Uncertainty in Multidisciplinary Design Optimization, AIAA2000-4803, Proceedings of $8^{\text {th }}$ AIAA/USAF/NASA/ISSMO Symposium on Multidisciplinary Analysis and Optimization, Long Beach, USA, (2000).

[2] N. Egorov, Optimization of a Multistage Axial Compressor. Stochastic Approach, ASME, 92-GT-163, (1992).

[3] N. Egorov and G. V. Kretinin, Search for Compromise Solution of the Multistage Axial Compressor's Stochastic Optimization Problem, World Publishing Corporation, Aerothermodynamics of internal flows III, Beijing, China, (1996), pp. 112-120.

[4] N. Egorov, G. V. Kretinin, I. A. Leshchenko, Y. I. Babiy, Optimization of complex engineering systems using variable-fidelity models, MCB University Press, ISBN: 0-86176-650-4, Proceedings of the 1st ASMO UK/ISSMO conference on Engineering Design Optimization, (1999), pp.143-149.

[5] N. Egorov, G. V. Kretinin, I. A. Leshchenko, Stochastic Optimization of Parameters and Control Laws of the Aircraft Gas-Turbine Engines - a Step to a Robust Design, Elsevier Science Ltd, "Inverse Problem in Engineering Mechanics III", (2002), pp.345...353.

[6] P. N. Koch, B. Wujek and O. Golovidov, A Multi-Stage, Parallel Implementation of Probabilistic Design Optimization in an MDO Framework, AIAA-2000-4805, Proceeding of $8^{\text {th }}$ AIAA/USAF/NASA/ISSMO Symposium on Multidisciplinary Analysis and Optimization, Long Beach, USA, (2000).

[7] Jacek Marczyk, Stochastic Multidisciplinary Improvement: Beyond Optimization, AIAA2000-4929, Proceedings of $8^{\text {th }}$ AIAA/USAF/NASA/ISSMO Symposium on Multidisciplinary Analysis and Optimization, Long Beach, USA, (2000). 
[8] M. T. Tong, A Probabilistic Approach to Aeropropulsion System Assessment, 2000-GT0001, Proceedings of ASME TURBOEXPO 2000,Munich, Germany, (2000).

[9] Ryohei Yokoyama and Koichi Ito, Robust Optimal Design of a Gas Turbine Cogeneration Plant Based on Minimax Regret Criterion, 99GT-128, Presented at the International Gas Turbine \& Aeroengine Congress \& Exhibition, Indianapolis, USA, (1999).

[10]Y-T. Wu, Methods for Efficient Probabilistic Analysis of Systems with Large Numbers of Random Variables, AIAA-98-4908, Proceeding of $7^{\text {th }}$ AIAA/USAF/NASA/ISSMO Symposium on Multidisciplinary Analysis and Optimization, St. Louis, USA, (1998). 